\title{
Relationship between Fructosamine Levels and Microalbuminuria of Selected Individuals with Type 2 Diabetes Mellitus
}

\author{
Gil P. Soriano, Ma. Gladys B. Aquino
}

Assistant Professor, School of Medical Technology, Centro Escolar University, Manila, Philippines

\begin{abstract}
:
Objectives: The research determined the relationship between the fructosamine level and microalbuminuria of selected individuals with Type 2 diabetes mellitus. The determination will suggest the effect of short-term glycemic (using fructosamine) to the impairment of kidney function (through the detection of microalbuminuria) due to kidney damage.
\end{abstract}

Methods: The study utilized descriptive correlational design to compare the fructosamine level and microalbuminuria. Colorimetric method using nitro blue tetrazolium was used to measure the fructosamine level from venous blood. In determining urine albumin level, immunoturbidimetric method was used; both blood and urine creatinine was measured using coupled enzymatic reactions.

Results: All of the selected participants had fructosamine levels above the normal value, which is 205-280 umol/L. Almost 50 percent of the participants were microalbuminuric (16 out of 32) and 50 percent (16 out of 32) were normoalbuminuric.

Conclusion: There is a significant difference between the fructosamine level of normoalbuminuric and microalbuminuric participants while there is no correlation between fructosamine levels and microalbuminuria.

Keywords: Diabetes Mellitus, Fructosamine, Normoalbuminuric, Microalbuminuric

\section{INTRODUCTION}

Diabetes mellitus is a chronic life-long condition characterized by poor glucose control. Globally, one person dies every seven seconds due to diabetes while an estimated 382 million individuals or a total of $8.3 \%$ of the world population are living with diabetes [1]. Moreover, in the Philippines, it is the eighth leading cause of death [2] and based on the recent study, almost $7.2 \%$ of its population is diagnosed with Type 2 diabetes mellitus [3].The disease entails a number of microvascular and macrovascular complications such as diabetic nephropathy. An early indicator of diabetic nephropathy is microalbuminuria or urinary excretion of 30 to 300mg of albumin per day [4] and among patients with type 2 diabetes, 20 to $40 \%$ of patients with microalbuminuria will progress to overt nephropathy, though only $20 \%$ of those patients will go on to end stage renal disease (ESRD) within the next 20 years [5].

Individuals with Type 2 diabetes mellitus must regularly monitor their serum blood glucose level in order to prevent these complications and one of the most common indicator for glucose control is Glycosylated Hemoglobin (HbA1c). It gives an integrated index of glycaemia over the entire 120-day lifespan of the red blood cells [6] and it is correlated with the development of long-term diabetic complications. However, results of the Atherosclerosis Risk in Communities study (ARIC) suggest that fructosamine could be useful as adjunctive tests when HbA1c might not be valid, or when assessing short-term glycemic control [7] and reflects the mean glucose levels over the recent two to three weeks.

The researchers determined the relationship between microalbuminuria and the fructosamine levels of selected individuals with Type 2 diabetes mellitus and the difference in the fructosamine levels of normoalbuminuric and microalbuminuric participants. 


\section{MATERials AND METHOD}

\subsection{Screening of Participants}

Fifty (50) individuals with Type 2 diabetes mellitus who sought consultation in an Out-Patient Department of a Tertiary Hospital were included in the study. The participants were selected based on the inclusion and exclusion criteria set by the researchers. The inclusion criteria include individuals with no diabetic complications, no comorbidity, and must be taking only oral hypoglycemic agent, insulin, antilipidemic drugs, and anti-hypertriglyceridemia. Individuals taking anti-inflammatory or immunesuppressive drug and patients with inflammation were excluded in the study.

\subsection{Ethical Consideration}

The study was approved by the Centro Escolar University ethics committee. Informed consents were gathered from the participants and anonymity were ensured throughout the conduct of the study.

\subsection{Collection of Sample}

Venous blood $(5 \mathrm{ml})$ samples were collected from the participants and serum was separated immediately. Sera was stored at $4^{0} \mathrm{C}$ until used. Random urine samples $(10 \mathrm{ml})$ were collected into clean containers provided by the researchers and were examined immediately.

\subsection{Sample Analysis}

Collected samples were submitted to a clinical laboratory for fructosamine, urine and serum creatinine, urine and serum albumin, and albumin-creatinine ratio assays. Colorimetric method using nitrobluetetrazolium (NBT) was used to measure the fructosamine level. In determining the albumin level, colorimetric assay was used for both serum and urine. For measuring serum and urine creatinine, the enzymatic method was utilized. Data obtained were analyzed using Pearson's $r$ and independent $t$ test with SPSS ver. 21.

\section{RESULTS}

Although 50 respondents passed the screening, only 32 passed the inclusion criteria. The other 18 participants were excluded due to presence of macroalbuminuria as shown in their urine albumincreatinine ratio, and serum and urine creatinine levels above the reference range denoting the presence of renal complication.

\subsection{Fructosamine Level}

As described, the level of fructosamine reflects the average of the continuously varying blood glucose concentrations in the blood for 2 to 3 weeks. In this study, there was a consistent hyperglycemia as none of the patients had fructosamine value within or below the normal range.

The computed mean fructosamine of all participants was $396.06 \mathrm{umol} / \mathrm{L}$, this value was 39-93 percent greater than the reference fructosamine level of 205-285 umol/L. More than half of the respondents (18 out of 32) had fructosamine level that are between the range of 321 to $390 \mathrm{umol} / \mathrm{L}$.

Table1. Profile of the Participants in terms of Fructosamine Level

\begin{tabular}{|c|c|c|}
\hline Fructosamine levels (umol/L) & Frequency $(\mathbf{n}=\mathbf{3 2})$ & Percentage \\
\hline $250-320$ & 5 & 15.63 \\
\hline $321-390$ & 17 & 53.13 \\
\hline $391-460$ & 3 & 9.38 \\
\hline $461-530$ & 3 & 9.38 \\
\hline $531-600$ & 2 & 6.25 \\
\hline $601-670$ & 1 & 3.13 \\
\hline $671-740$ & 1 & 3.13 \\
\hline
\end{tabular}

\subsection{Albumin-creatinine Ratio}

Among the 32 participants, 16 were normoalbuminuric while the other 16 were microalbuminuric. Normoalbuminuric participants had urine albumin-creatinine ratio of $0-29.9 \mathrm{mg} / \mathrm{g}$. While microalb- 
uminuric participants had urine albumin-creatinine ratio of $30-300 \mathrm{mg} / \mathrm{g}$. The mean urine albumincreatinine ratio of the 32 participants was $54.3 \mathrm{mg} / \mathrm{g}$. It is 81.61 percent greater than the normal urine albumin-creatinine ratio of $0-29.9 \mathrm{mg} / \mathrm{g}$.

Table2. Profile of the Participants in terms of albumin-creatinine ratio

\begin{tabular}{|c|c|c|}
\hline Albumin-creatinine ratio $(\mathrm{mg} / \mathrm{g})$ & Frequency & Percentage \\
\hline $0-29.9$ & 16 & 50.0 \\
\hline $30-74.9$ & 8 & 25.0 \\
\hline $75-119.9$ & 4 & 12.5 \\
\hline $120-164.0$ & 1 & 3.13 \\
\hline $165-209.9$ & 1 & 3.13 \\
\hline $210-254.9$ & 1 & 3.13 \\
\hline $255-300$ & 1 & 3.13 \\
\hline
\end{tabular}

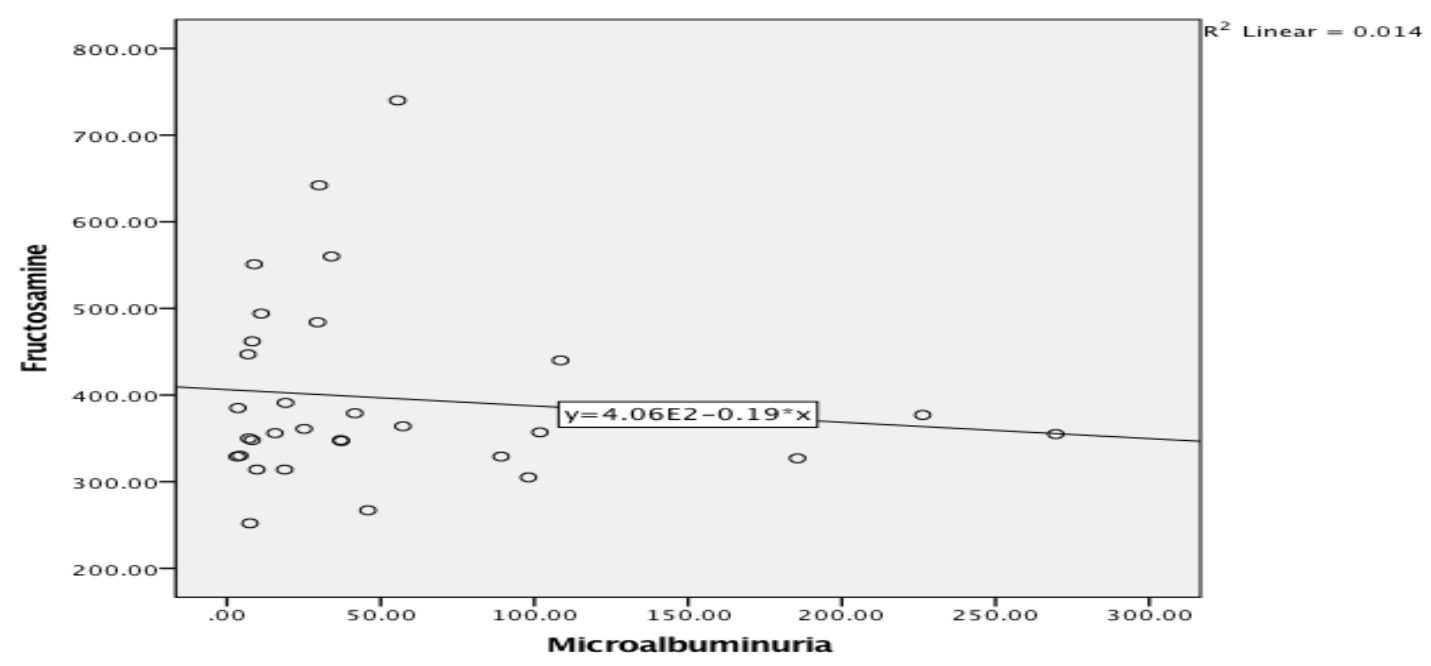

Figure1. Scatterplot of Fructosamine Levels versus albumin-creatinine ratio

\section{DISCUSSION}

Fructosamine is a ketoamine formed in a two-step process involving the reaction of the carbonyl group of glucose to the amino group of protein while the second step involves the transformation of the nonreversibleAmadori rearrangement to the corresponding ketoamine. The ketoamine formed is fructosamine and is considered the most used alternative to HbAlc. Fructosamine is considered a reliable indicator of glycemic control $[8,9]$, however, it was reported that the value of fructosamine in clinical practice remains unclear [10].

In order to derive the desired albumin-creatinine ratio, both urine albumin and urine creatinine were measured. Although urine albumin alone can diagnose microalbuminuria, urine creatinine was also measured to avoid false results brought by natural variations in protein concentration as well as in cases of dehydration. In this manner, creatinine is known to be excreted on a regular and constant basis. Using this measure of creatinine, in comparison with albumin whose excretion rate varies, in the same urine specimen gives a more reliable comparison of results in terms of kidney function. Meanwhile, serum albumin and serum creatinine were also measured to counter check the values obtained from the urine specimen.

The gathered data revealed that among the 32 participants, 50 percent were microalbuminuric (16 out of 32) while the remaining 50 percent (16 out of 32) were normoalbuminuric. Normoalbuminuric participants are Type 2 Diabetes Mellitus patients whose urine albumin are below $29.9 \mathrm{mg}$ per day. While microalbuminuric participants are Type 2 Diabetes Mellitus patients whose urine albumin exceeded the normal value thereby falling between the ranges of 30-300 mg per day. Normoalbuminuric patients served as the control variable in the study. 
The mean fructosamine of normoalbuminuric participants was $385.5 \mathrm{umol} / \mathrm{L}$ while the mean fructosamine of microalbuminuric participants was $406.6 \mathrm{umol} / \mathrm{L}$. This shows that microalbuminuric participants had higher fructosamine level by about 5.20 percent than normoalbuminuric participants. The computed pvalue of 0.000 , using the Welch t-test, revealed a significant difference between the fructosamine level of normoalbuminuric and microalbuminuric participants.

Baseline characteristics of participants were compared in terms of fructosamine level and their degree of microalbuminuria. The relationship between fructosamine from serum and microalbuminuria were compared using Pearson product-moment correlation coefficient. The computed $p$ value of 0.517 revealed no correlation between fructosamine levels and microalbuminuria of the 32 selected Type 2 Diabetes Mellitus participants. (Figure 1) Factors such asconsistent high fructosamine level and one-time sample collection may have an effect on the obtained data.

\section{Conclusions}

The study concludes that a significant difference exists between the fructosamine level of normoalbuminuric and microalbuminuric participants. However, there is no significant correlation between fructosamine levels and microalbuminuria.

\section{REFERENCES}

[1] International Diabetes Federation (2014). IDF Diabetes Atlas, $6^{\text {th }}$ ed.; 11-17.

[2] Department of Health (2014, October 10). Leading Causes of Mortality. Retrieved from http://www.doh.gov.ph/node/2573

[3] Jimeno, C., Kho, S., Matawaran, B., Durante, C. and Jasul, G. Prevalence of Diabetes Mellitus and Pre-Diabetes in the Philippines: A Sub-study of the 7th National Nutrition and Health Survey. Philippine Journal of Internal Medicine. 53 (2), 2015

[4] Evans, T., and Capell, P. Diabetic Nephropathy. Clinical Diabetes, 18 (1), 2000

[5] 1American Diabetes Association: Position statement: Diabetic nephropathy. Diabetes Care 22 (Suppl 1): S66-69, 1999

[6] Tahara Y., and Shima K. Kinetics of HbAlc, glycated albumin, and fructosamine and analysis of their weight functions against preceding plasma glucose level. Diabetes Care. 18:440-447, 1995

[7] Selvin E., Rawlings A., and Grams M. Fructosamine and glycated albumin for risk stratification and prediction of incident diabetes and microvascular complications: a prospective cohort analysis of the Atherosclerosis Risk in Communities (ARIC) study. Lancet Diabetes Endocrinol 2: 279-288, 2014

[8] Baker J., Metcalf P., Holdaway I., and Johnson R. Serum fructosamine concentration as measure of blood glucose control in type I (insulin-dependent) diabetes mellitus. Br Med J 290: 352-355, 1985a.

[9] Baker J, Reid I, Holdaway I: Serum fructosamine in patients with diabetes mellitus. N Z Med J 98: 532-535, 1985b.

[10] Jerntorp P, Sundkvist G, Fex G, Jeppsson J.: Clinical utility of serum fructosamine in diabetes mellitus compared with hemoglobin Alc. Clin Chim Acta 175: 135-142, 1988. 